\title{
Blockade of Notch3 inhibits the stem-like property and is associated with ALDH1A1 and CD44 via autophagy in non-small lung cancer
}

\author{
YUANYUAN MA ${ }^{1}$, MINGZHEN LI ${ }^{2}$, JIAHUI SI ${ }^{1}$, YING XIONG ${ }^{1}$, FANGLIANG LU ${ }^{1}$, \\ JIANZHI ZHANG ${ }^{1}$, LIYI ZHANG ${ }^{1}$, PANPAN ZHANG ${ }^{1}$ and YUE YANG $^{1}$ \\ ${ }^{1}$ Key Laboratory of Carcinogenesis and Translational Research (Ministry of Education/Beijing), \\ Department of Thoracic Surgery II, Peking University Cancer Hospital and Institute, Beijing 100142; \\ ${ }^{2}$ Beijing Center for Physical and Chemical Analysis, Beijing 100089, P.R. China
}

Received November 28, 2015; Accepted December 31, 2015

DOI: $10.3892 /$ ijo.2016.3464

\begin{abstract}
Acquired resistance to standard chemotherapy causes treatment failure in patients with local advanced and advanced non-small lung cancer (NSCLC). Cancer stem cells (CSCs) are a small subpopulation within cancer that is thought to be resistant to conventional chemotherapy. The Notch pathway is one of the most intensively studied for putative therapeutic targets of CSCs in solid tumors. In our study, suppression of Notch3 decreased colony and sphere formation of stem-like property in lung cancer cells. In addition, Notch3 expression was demonstrated to be upregulated in the patients with chemoresistance and related to poor prognosis of NSCLC patients. Our results also showed that CSC markers ALDH1A1 and CD44 were highly expressed in NSCLC patients with chemoresistance and these two markers were positively correlated with Notch3 expression in lung cancer specimens from TCGA database. Furthermore, the lung cancer cells with drug resistance were shown to be associated with activation of autophagy. All the data support a crucial role of Notch3 in the increase of stem-like property in NSCLC cells that might be associated with upregulation of ALDH1A1 and CD44 and activation of autophagy.
\end{abstract}

\section{Introduction}

Lung cancer is the leading cause of cancer-related deaths worldwide (1). Non-small cell lung cancer (NSCLC), with the two major pathological subtypes of adenocarcinoma (Ad) and squamous cell carcinoma (SCC) accounts for approximately $85 \%$ of all lung cancers. NSCLC patients have low overall survival (OS) rate, and there has been little change in the OS

Correspondence to: Dr Yue Yang, Department of Thoracic Surgery II, Peking University Cancer Hospital and Institute, 52 Fucheng Road, Haidian District, Beijing 100142, P.R. China E-mail: zlyangyue@bjmu.edu.cn

Key words: Notch3, cancer stem cells, non-small cell lung cancer, ALDH1A1, CD44 rate over the past three decades (1). Chemotherapy can be used as the primary treatment for advanced NSCLC or as an adjuvant treatment after surgery for the patients with lymph node metastasis. Initially chemotherapy has efficacy in the NSCLC patients, but chemoresistance finally occurs (2).

Cancer stem cells (CSCs) are defined as a small subpopulation, showing properties of self-renewal, differentiation, tumorigenesis and drug resistance (3). CSCs have been documented in several types of cancers including breast, prostate, colon, and lung cancers (4-7). Recent studies have provided evidence that CSCs are responsible for the poor prognosis of lung cancer patients and contribute to chemoresistance for the lung cancer cells (8-10). CD133, CD44, ALDH1A1, CD90, and CD166 were verified to be effectively used as CSC markers in NSCLC (11-13). Notch pathway has been implicated in CSC control and cell fate determination (14). In mammals, there are four Notch receptors Notch1, Notch2, Notch3 and Notch4. Notch interacts with its ligand that give rise to release of Notch intracellular domain (NICD). NICD then translocates to the nucleus to regulate target gene transcription $(15,16)$. It has been reported that high activation of Notch was associated with poor outcome for lung cancer patients (17). Notch3 has been found to be associated with lung CSCs and is a potential therapeutic target in lung cancer (18). In addition, when Notch3 expression was knocked down using small interfering RNA (siRNA), the proliferation was also reduced in lung cancer cells (19).

However, how Notch3 influences CSCs and regulate chemoresistance in the lung cancer cells are not fully elucidated. In the current study, cisplatin was found to induce clonogenicity of stem-like property and this effect could be inhibited when Notch3 was knocked down in the lung cancer cells. The Notch3 overexpression was associated with poor outcome of NSCLC patients. CSC markers CD44 and ALDH1A1 were shown to be positively related to Notch3 expression. Particularly, $\gamma$-secretase inhibition (GSI) could suppress stem-like features in lung cancer cells with chemoresistance and also led to decreased expression levels of CD44 and ALDH1A1. Moreover, autophagy was involved in cisplatin resistance of lung cancer that might be through Notch3 signal. 


\section{Materials and methods}

NSCLC patients. Frozen tissues were obtained from 104 NSCLC patients surgically resected for stage I-IIIA NSCLC at Peking University Cancer Hospital, from 2006 to 2009. The formalin-fixed tissues derived from NSCLC patients who received platinum-based neoadjuvant chemotherapy were studied in the current investigation. The study was carried out in accordance with the approved guidelines of the local ethics committee of Peking University Cancer Hospital, and informed consent was obtained from all the subjects.

Immunohistochemistry (IHC). IHC was carried out on formalin-fixed sections (4-mm). After deparaffination of the sections, endogenous peroxidase activity was blocked by $3 \%$ $\mathrm{H}_{2} \mathrm{O}_{2}$ solution for $10 \mathrm{~min}$. Then, antigen retrieval was achieved by microwave in citrate buffer $(\mathrm{pH}$ 6.0) followed by blocking in 5\% goat serum in PBS for $15 \mathrm{~min}$. The primary antibodies including Notch3 (Santa Cruz Biotechnology, sc-5593, 1:400 dilution), CD44 (Abcam, ab51037, 1:400 dilution) and ALDH1A1 (Abcam, ab52492, 1:400 dilution) were used to incubate the sections at $4^{\circ} \mathrm{C}$ overnight. HRP-conjugated secondary antibody was added on the sections for $30 \mathrm{~min}$. Then, substrate-chromogen (DAB) solution was employed to incubate the tumor tissues for $10 \mathrm{~min}$ and automated hematoxylin was used to finally counterstain the slides for $5 \mathrm{~min}$. The immunostaining was microscopically evaluated by two independent pathologists.

Cell culture and cell treatment. Human NSCLC cell lines A549 and H520 were maintained in the RPMI-1640 medium (Hyclone) containing 10\% fetal bovine serum (FBS) and 1\% penicillin/streptomycin in a humidified $5 \% \mathrm{CO}_{2}$ incubator at $37^{\circ} \mathrm{C}$. A549 and H520 cells were treated with GSI $(5 \mu \mathrm{g} / \mathrm{ml})$ obtained from Sigma-Aldrich Corp. Cisplatin ( $1 \mu \mathrm{g} / \mathrm{ml})$ (Qilu Pharmaceutical, China) was added to treat the A549 and H520 cells for $72 \mathrm{~h}$ and the cells were recovered in normal medium for additional $24 \mathrm{~h}$.

Colony formation assay. The single cells were prepared and planted into the 6-well plates. After 10 days, the cells were fixed with $4 \%$ formaldehyde for $15 \mathrm{~min}$ followed by PBS washing. After that, the cells were stained with crystal for 15 min and the cells were washed with PBS at least twice. The colonies were evaluated by inverse microscopy. Colony formation efficiency was determined as: colony formation efficiency $=$ colony/input cells $\times 100 \%$.

Sphere formation assay. The cells were digested and the single cells were seeded in ultralow attachment 96-well plates (Corning Inc., Life Sciences). The DMEM/F-12 (Invitrogen) serum-free medium including B27 (Invitrogen), $20 \mathrm{ng} / \mathrm{ml}$ epidermal growth factor (Peprotech, Rocky Hill, NJ, USA), $20 \mathrm{ng} / \mathrm{ml}$ basic fibroblast growth factor (Peprotech), $10 \mathrm{ng} / \mathrm{ml}$ hepatocyte growth factor (Peprotech), and $1 \%$ methylcellulose (Sigma) were used to cultivate the cells for 10 days. The formed spheres were counted by inverse microscopy. Sphere formation efficiency was calculated as: sphere formation efficiency $=$ sphere/input cells $\times 100 \%$.
Transient transfection. The specific siRNA834 and siRNA3408 to know down Notch3 were obtained from Suzhou GenePharma Corp., China. The siRNA834 and siRNA3408 and scramble siRNA were transfected to the A549 and H520 cells by X-tremeGENE siRNA transfection reagent according to the manufacturer's instructions (Roche). After $48 \mathrm{~h}$, the cells were collected for further experiments.

Western blotting. Cell pellets were lysed in RIPA lysis buffer added with phenylmethylsulfonylfluoride (PMSF), Complete Mini protease inhibitor cocktail, and phosphatase inhibitor cocktail (Roche). The protein was measured and equal amount of protein was separated on a $10 \%$ SDS-PAGE gel, and then transferred to nitrocellulose membrane (Millipore). $5 \%$ fat-free milk was used to block the membrane, and primary antibodies of Notch3 (Santa Cruz Biotechnology, sc-5593, 1:500 dilution), LC3 (MBL International Corp., 1:1000 dilution) and GAPDH (Cell Signaling Technology, \#2118, 1:5000 dilution) were added on the membrane for $1 \mathrm{~h}$ at room temperature. After washing three times in Tris Buffered Saline plus Tween (TBST), the membranes were added with the corresponding secondary antibody conjugated with HRP. The signal was visualized using Immobilon Western Chemiluminescent HRP Substrate (Millipore).

Flow cytometry. The lung cancer cells A549 and H520 were washed in PBS and collected by centrifrugation. The cells were stained with primary antibodies including Notch3 (Santa Cruz Biotechnology, sc-5593, 1:50 dilution), CD44 (Abcam, ab51037, 1:100 dilution) and ALDH1A1 (Abcam, ab52492, 1:100 dilution) at $4^{\circ} \mathrm{C}$ for $1 \mathrm{~h}$. After washing with PBS twice, the cell suspension was incubated with secondary antibody conjugated with FITC at $4^{\circ} \mathrm{C}$ for $30 \mathrm{~min}$ in the dark. The cells were filtered through a $70-\mu \mathrm{m}$ nylon mesh and carried out on a flow cytometer (BD Biosciences). The viable and single cells were gated for analyses. FlowJo 7.6 software was used to analyze the data.

RNA extraction and quantitative real time-PCR ( $q R T-P C R)$. Total RNA was isolated from human NSCLC tissues using TRIzol Reagent (Invitrogen) according to the manufacturer's instructions. Total RNA $(2 \mu \mathrm{g})$ was reverse-transcribed to cDNA with Moloney murine leukemia virus (MMLV, Invitrogen). The genes expression levels were measured by qRT-PCR with the SYBR Green PCR kit (Roche) using Light-Cycler ${ }^{\circledR} 480$ Real-Time PCR System (Roche). The reactions were incubated in a 96 -well optical plate at $95^{\circ} \mathrm{C}$ for $10 \mathrm{~min}$, followed by 40 cycles of $95^{\circ} \mathrm{C}$ for $15 \mathrm{sec}$ and $60^{\circ} \mathrm{C}$ for $1 \mathrm{~min}$. $\beta$-actin was used as internal control and Notch3 expression level was calculated according to $2^{-\Delta \mathrm{Ct}}$, in which $\Delta \mathrm{Ct}=\mathrm{Ct}$ target $-\mathrm{Ct}$ control. All PCR assays were carried out in triplicate, and the mean of triplicates is reported.

Immunofluorescence staining. Briefly, the cells were washed with PBS and fixed in cold $10 \%$ paraformaldehyde. The cells were washed twice in PBS and incubated with the primary antibody LC3 (MBL International Corp., 1:100 dilution) at $4^{\circ} \mathrm{C}$ overnight. After washing with PBS, the cells were added with secondary antibody at room temperature for $1 \mathrm{~h}$. Finally, slides were washed with PBS, mounted with DAPI in mounting 
A

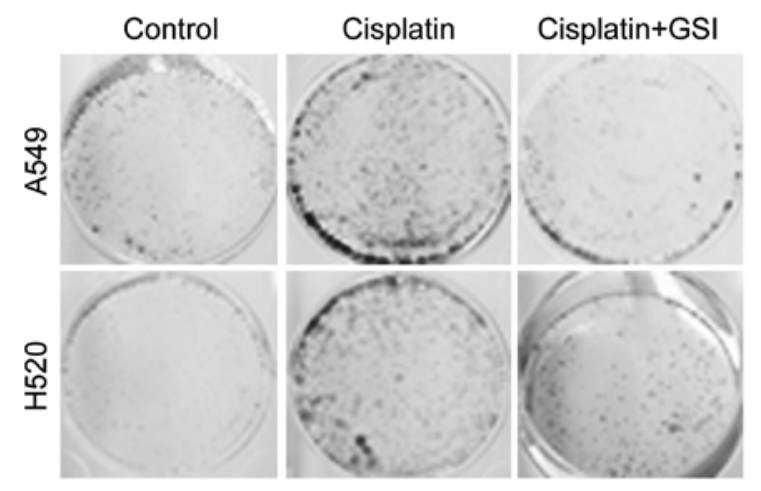

C

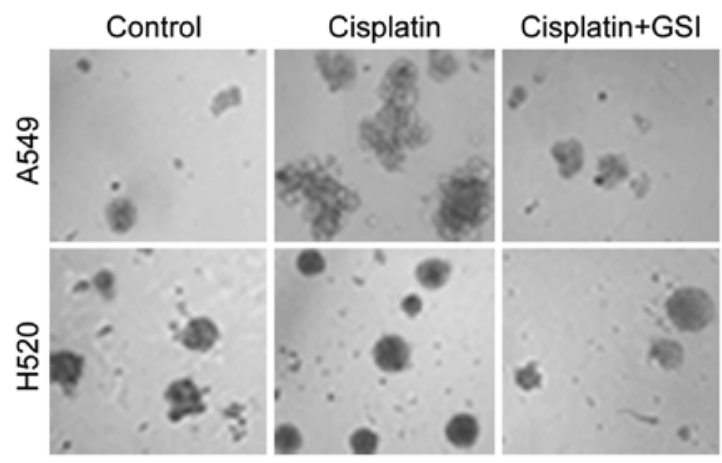

B

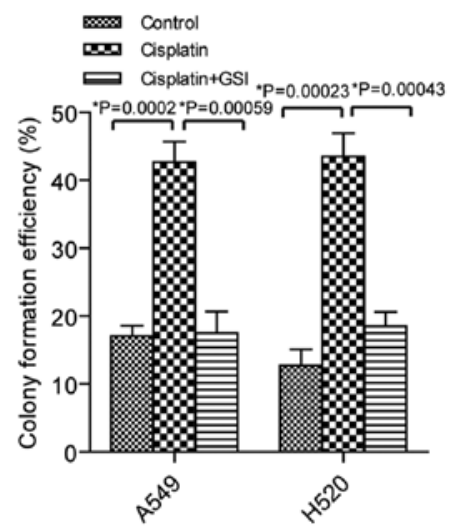

D

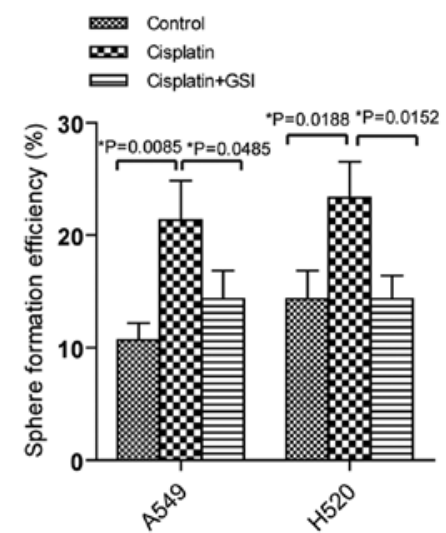

Figure 1. Colony and sphere formation analyses. (A) Representative images for the colony formation in A549 and H520 cells with cisplatin or cisplatin plus GSI. (B) Histograms show that cisplatin increases colony formation frequencies, while GSI inhibited colony number to similar level of control cells with significant difference in both A549 and H520 cells. (C) Representative images for the sphere formation in the control cells, cisplatin treated cells and cisplatin plus GSI treated cells of A549 and H520. (D) Histograms demonstrate that cisplatin upregulates sphere formation efficiency, but GSI inhibited sphere number to similar level of control cells with significant difference in both A549 and H520 cells. * p<0.05.

media. Cells were examined using a laser scanning confocal microscope (Leica, Wetzlar, Germany).

Statistical analyses. Data are shown as mean \pm SEM and the experiments were independently done at least three times. Student's t-test was used to analyze the mean between different groups. Spearman's correlation test was carried out to assess correlation between Notch3 and CSC markers expression. Survival curves were analyzed by the log-rank Kaplan-Meier method. GraphPad Prism 5 was conducted for data analysis and $\mathrm{p}<0.05$ was considered to indicate a statistically significant difference.

\section{Results}

Cisplatin promotes clonogenicity in A549 and H520 cells. It has been reported that CSCs have ability to be related to drug resistance in lung cancer (2). We investigated how cisplatin as first-line chemotherapy influences cell clonogenicity of stemlike property. To obtain drug resistant cells, the lung cancer cells A549 and H520 were treated with cisplatin for $72 \mathrm{~h}$ and the dead cells were removed by washing in PBS. After that, the single cells were seeded into 6-well plates or low attachment plates for further cultivation. Ten days later, more colonies were observed in the lung cancer cells with cisplatin treatment than the control cells with significant statistical difference in both A549 and H520 cells (Fig. 1A and B p<0.05). In addition, more spheres were formed in the A549 and H520 cells stimulated with cisplatin compared to the cells without cisplatin (Fig. 1C and D, p<0.05). These data suggested that cisplatin induced clonogenicity of stem-like ability in lung cancer cells.

Inhibition of Notch3 suppresses clonogenicity in A549 and H520 cells. GSI was reported to prevent Notch3 activation and reduce proliferation in human lung cancers (19). GSI was added in A549 and H520 cells before cisplatin treatment, and then colony and sphere formation were examined. As shown in Fig. 1A and B, colonies were reduced in the cells with cisplatin plus GSI compared to the cells with cisplatin. Similarly, decreased sphere number was identified in the cells stimulated with cisplatin plus GSI than the cells treated with cisplatin, showing statistically significant difference (Fig. 1C and D).

We have mainly studied the role of Notch3 in regulating stem-like ability in lung cancer cells of A549 and H520. Thus, we used specific siRNAs to knock down Notch3 expression in these lung cancer cells. Firstly, the siRNA834 and siRNA3408 
A

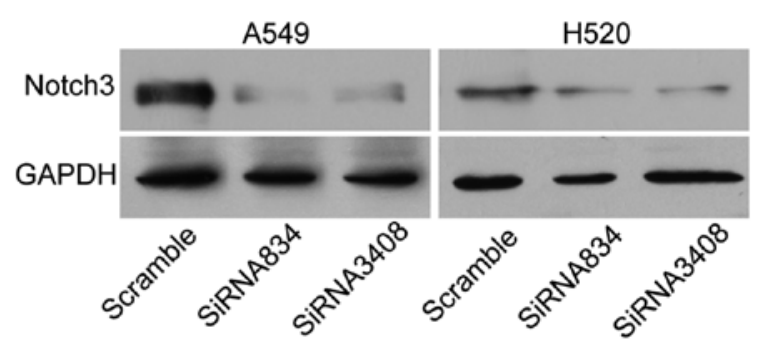

B

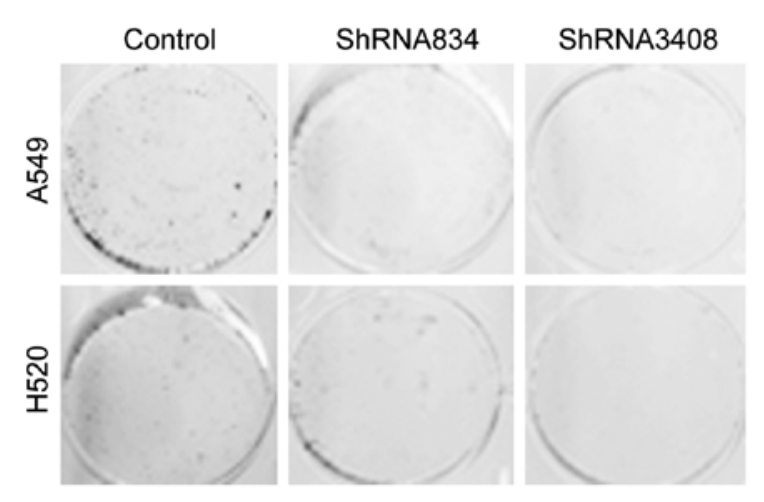

C

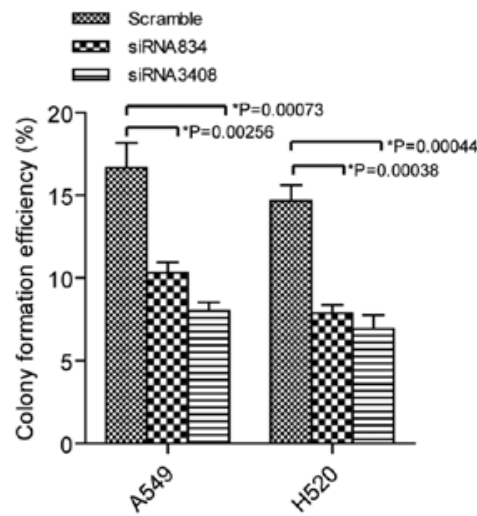

D

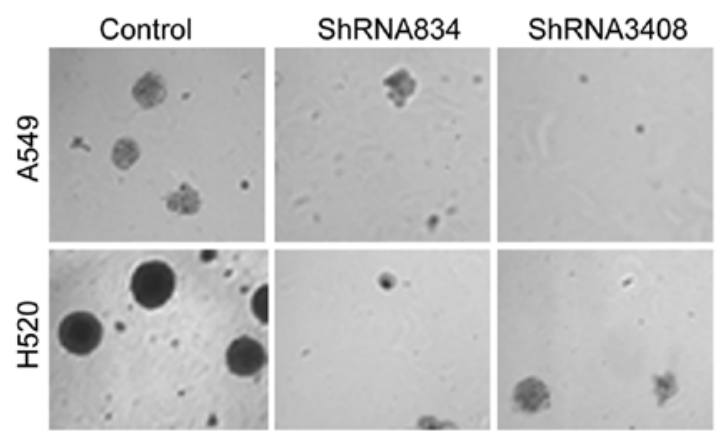

$\mathrm{E}$

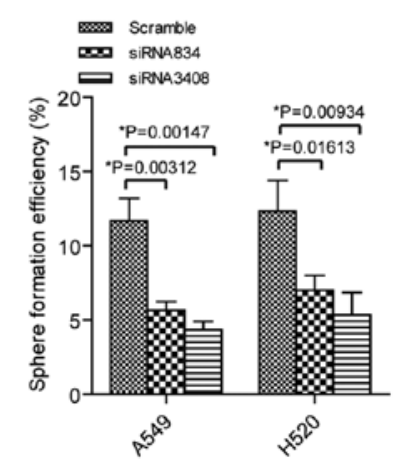

Figure 2. Colony and sphere formation in A549 and H520 cells transfected with scramble siRNA, siRNA834 and siRNA3408. (A) Notch3 expression was decreased in the lung cancer cells transfected with siRNA834 and siRNA3408 by western blot examination. (B) Representative photographs for the colony formation in A549 and H520 cells transfected with scramble siRNA, siRNA834 and siRNA3408. (C) SiRNA834 and siRNA3408 reduced the colony number with significant difference in the lung cancer cells. (D) Representative photographs for the sphere formation in A549 and H520 cells transfected with scramble siRNA, siRNA834 and siRNA3408. (E) Knock-down of Notch3 led to decreased sphere formation efficiency with significant difference in A549 and H520. ${ }^{*} \mathrm{p}<0.05$.

were used to knock down Notch3 expression in A549 and H520 cells and lower expression of Notch3 was found in these cells by western blotting (Fig. 2A). Next, clonogenic ability was tested in the cells transfected with scramble siRNA, siRNA834 or siRNA3408. As we expected, colony formation was decreased in the lung cancer cells with siRNA834 or siRNA3408 treatment compared to the cells with scramble siRNA treatment (Fig. 2B and C). Moreover, sphere formation was downregulated in the A549 and H520 cells transfected with siRNA834 and siRNA3408 compared to the cells with scramble control (Fig. 2D and E). Altogether, our data indicated that blocking of Notch 3 was able to inhibit stem-like features in lung cancer cells.
Notch 3 is associated with CD44 and ALDH1A1. Next, we identified the correlation between Notch 3 and the CSC markers CD44, ALDH1A1, KIT, CD133, EPCAM, CD166, CD90 and CD24 in lung cancer tissues from RNA-seq of TCGA database (https://tcga-data.nci.nih.gov/tcga/, $\mathrm{n}=349$ ). The analysis showed that Notch3 expression was positively related to CD44 and ALDH1A1 expression levels with statistically significant difference (Fig. 3A). Then, we tested CD44 and ALDH1A1 alterations when the Notch3 was knocked down in the lung cancer cells. Silenced of Notch3 resulted in downregulation of CD44 in A549 cells transfected with siRNA834 $(2.91 \pm 0.52 \%)$ and siRNA3408 $(2.09 \pm 0.37 \%)$ compared to the control cells $(5.69 \pm 0.28 \%)$, (Fig. 3B and C). H520 cells showed 
A

\begin{tabular}{|c|c|c|}
\hline \multicolumn{3}{|c|}{$\begin{array}{c}\text { orrelation between Notch3 and cancer stem cell } \\
\text { related genes in TCGA database }(\mathrm{n}=349)\end{array}$} \\
\hline Notch3 vs. & $\mathrm{P}$ & $\mathrm{R}$ \\
\hline CD44 & $9.76 \mathrm{E}-08$ & 0.281793 \\
\hline ALDH1A1 & $4.19 \mathrm{E}-05$ & 0.217956 \\
\hline KIT & 0.00042 & -0.18811 \\
\hline CD133 & 0.00101 & -0.17548 \\
\hline EPCAM & 0.028006 & -0.11766 \\
\hline CD166 & 0.035449 & 0.112655 \\
\hline CD90 & 0.046637 & 0.106597 \\
\hline CD24 & 0.055932 & -0.10243 \\
\hline
\end{tabular}

B

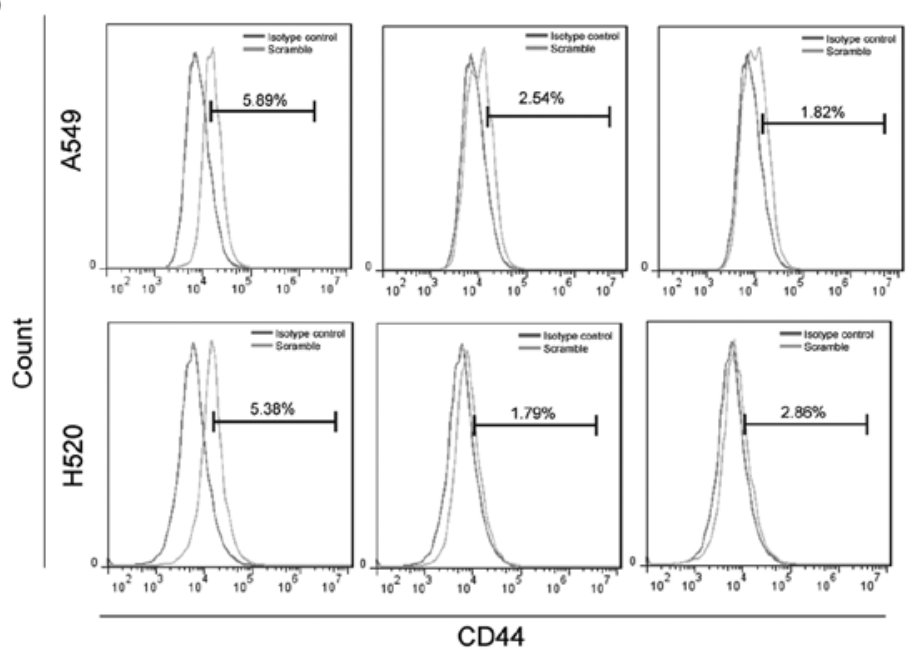

D

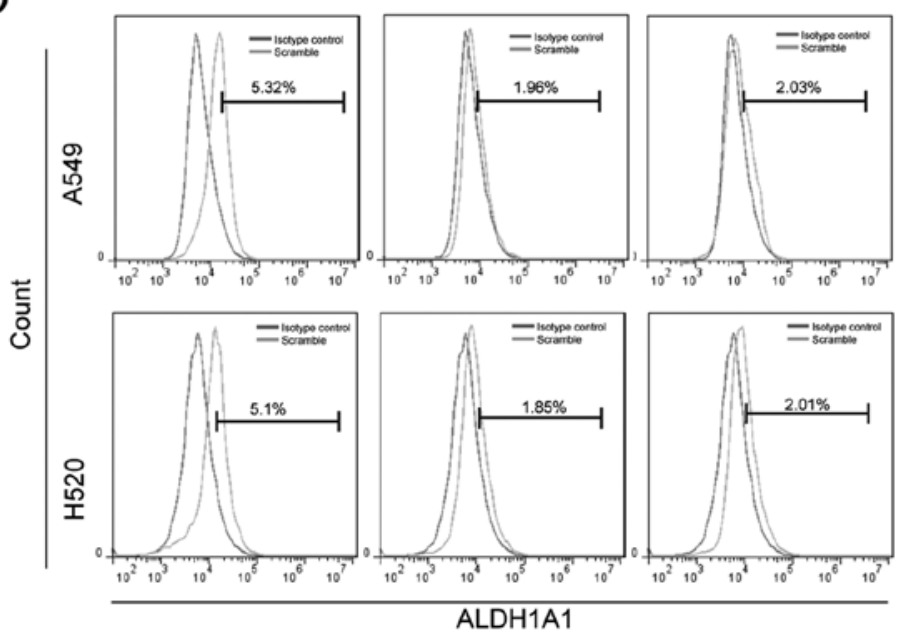

C

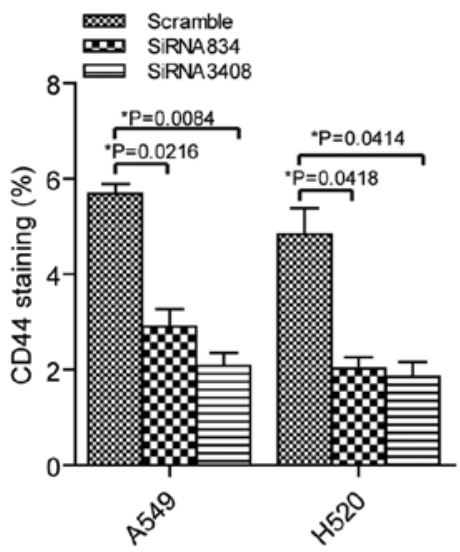

E

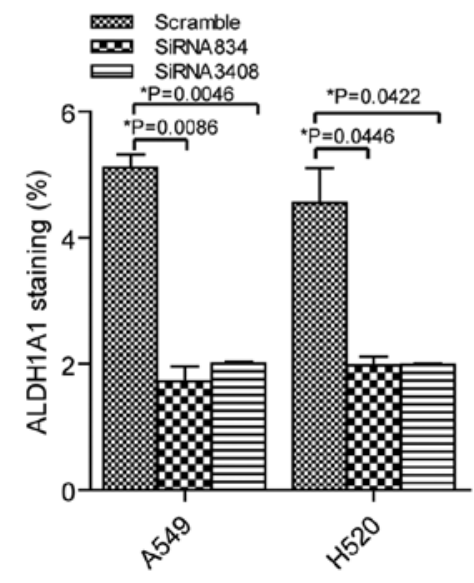

Figure 3. Relationship between Notch3, CD44 and ALDH1A1 expression levels. (A) Correlations between Notch3 and CSC markers including CD44, ALDH1A1, KIT, CD133, EPCAM, CD166, CD90 and CD24 were analyzed in the RNA-seq data of NSCLC tissues from TCGA. (B) Representative images for CD44 expression in the A549 and H520 cells with inhibition of Notch3 expression by flow cytometry test. (C) The data show that CD44 expression was reduced in the cells with siRNA834 and siRNA3408 transfection compared to the control cells. (D) Representative pictures for ALDH1A1 expression in the A549 and H520 cells transfected with scramble siRNA, siRNA834 and siRNA3408 by flow cytometry. (E) The results show decreased expression of ALDH1A1 in the lung cancer cells with siRNA834 and siRNA3408 than the cells with scramble siRNA. "p<0.05.

decreased expression of CD44 in siRNA834 transfected cells $(2.03 \pm 0.33 \%)$ and siRNA3408 transfected cells $(1.85 \pm 0.44 \%)$ than the cells with scramble siRNA $(4.83 \pm 0.77 \%)$. ALDH1A1 expression was reduced when Notch3 expression was blocked 
A

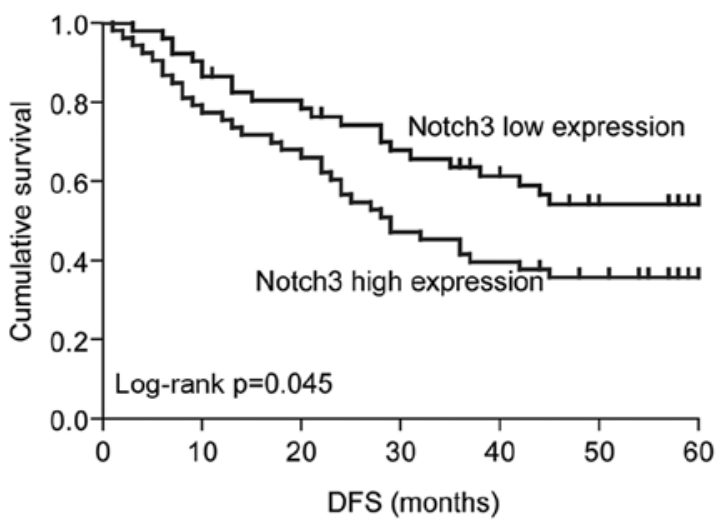

B

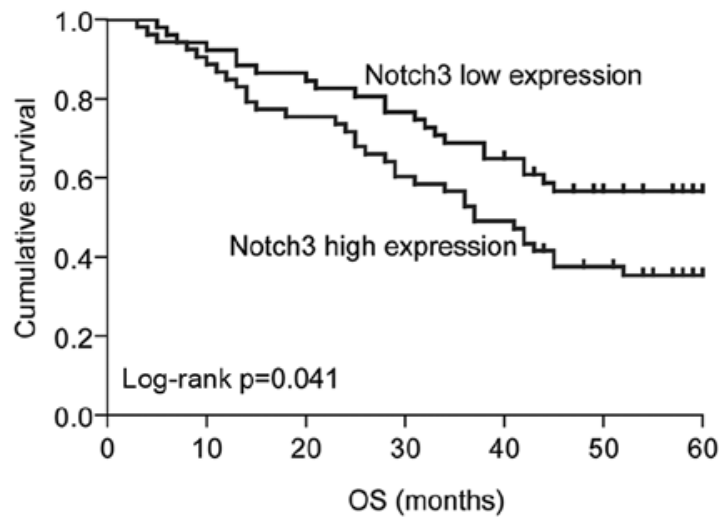

Figure 4. Notch3 as a predictor for poor outcome of NSCLC patients. (A) DFS curve according to Notch3 expression in 104 NSCLC patients. (B) OS curve according to Notch3 expression in 104 NSCLC patients.

in A549 cells transfected with siRNA834 (1.73 $\pm 0.33 \%)$ and siRNA3408 $(2.01 \pm 0.04 \%)$ compared to the control cells $(5.11 \pm 0.3 \%)$, (Fig. 3D and E). In addition, there was decreased expression of ALDH1A1 in siRNA834 transfected H520 cells $(1.99 \pm 0.19 \%)$ and siRNA3408 transfected cells $(1.99 \pm 0.04 \%)$ compared to the cells with scramble siRNA (4.56 $\pm 0.77 \%)$. Our results suggested that Notch3 was positively associated with CD44 and ALDH1A1 in lung cancer cells.

Notch3 is a prognostic factor. Since our data indicated that Notch3 promoted stem-like property, we tested the relationship between Notch3 expression and progression-free survival (DFS) and OS. Notch3 expression was investigated by qPR-PCR in the primary tumor tissues from 104 cases of NSCLC patients. According to the median value, Notch3 expression levels were divided into low expression $(n=52)$ and high expression $(n=52)$ in these NSCLC cases. Thus, we verified that high expression of Notch3 was associated with shorter PSF (median PSF in low expression group, 54.89 months and median PSF in high expression group, 42.11 months, $\mathrm{p}=0.045$ ) and OS (median OS in low expression group, 58.09 months and median OS in high expression group, 46.23 months, $\mathrm{p}=0.041$ ), (Fig. 4A and B). The results suggested that Notch3 may be used as a prognostic factor in NSCLC patients.

Cisplatin induces Notch3, CD44 and ALDH1A1 expression levels and GSI suppresses this induction in A549 and H520 cells. Then, we investigated Notch3, CD44 and ALDH1A1 expression in 12 NSCLC patients who received neoadjuvant chemotherapy. For Notch3 expression, there were 4 out of 6 cases with chemoresistance showing positive expression and 1 out of 6 cases with chemosensitivity demonstrating positive staining by IHC assay (Fig. 5A). Among 6 NSCLC patients with chemoresistance, 5 patients displayed positive staining for CD44 and ALDH1A1 expression (Fig. 5A). Whereas, there were 3 cases with positive expression of CD44 and 2 cases with positive staining of ALDH1A1 in 6 NSCLC patients with chemosensitivity as assessed by IHC analysis (Fig. 5A).

Moreover, we investigated how Notch3, CD44 and ALDH1A1 altered in the cisplatin treatment in A549 and H520 cells. Cisplatin led to 2.64-fold increase of Notch3 expression in A549 cells and 3.12-fold increase of this protein in H520 cells (Fig. 5B and C). Again, GSI was used to inhibit the Notch3 signal in the cells with cisplatin treatment. We observed that Notch3 expression was decreased in A549 and H520 cells with cisplatin plus GSI compared to this protein expression in the cells with cisplatin treatment. Similarly, increased expression levels of CD44 and ALDH1A1 were observed in the lung cancer cells treated with cisplatin compared to the cells without cisplatin treatment (Fig. 5D-G). There were 2.67-fold increase of CD44 in A549 cells treated with cisplatin and 1.98-fold increase of CD44 in H520 cells with cisplatin stimulation. In addition, we obtained 2.48-fold increase of ALDH1A1 in cisplatin-treated A549 cells and 2.3-fold increase of ALDH1A1 in cisplatin-treated H520 cells. CD44 and ALDH1A1 were downregulated in the cells treated with both cisplatin and GSI compared to the cells administered with cisplatin (Fig. 5D-G).

Cisplatin induces autophagy, and blockade of Notch3 suppresses this effect in A549 cells. Since autophagy is an adaptive response to a conventional chemotherapy and radiation therapy (20), we determined how autophagy changed in lung cancer cells with cisplatin treatment. It has been reported that the cells undergo autophagy, LC3I expression decreased and LC3II expression increased (21). Then, we tested LC3II expression in A549 cells with or without cisplatin treatment by the methods of western blotting and immunofluorescence. The results showed increase of LC3II in A549 cells with cisplatin treatment compared to the control cells (Fig. 6A and B). Next, we asked whether autophagy was associated with Notch 3 in the lung cancer cells. When Notch3 was blocked, LC3II expression was downregulated in the cells as evaluated by western blot and immunofluorescence analyses (Fig. 6C and D).

\section{Discussion}

Currently, chemoresistance is considered as a major obstacle for the clinical treatment of advanced and metastatic NSCLC patients. It is well known that CSCs have a capacity to escape from chemotherapy and radiotherapy. However, the mechanisms for the involvement of CSCs in chemoresistance have 
A

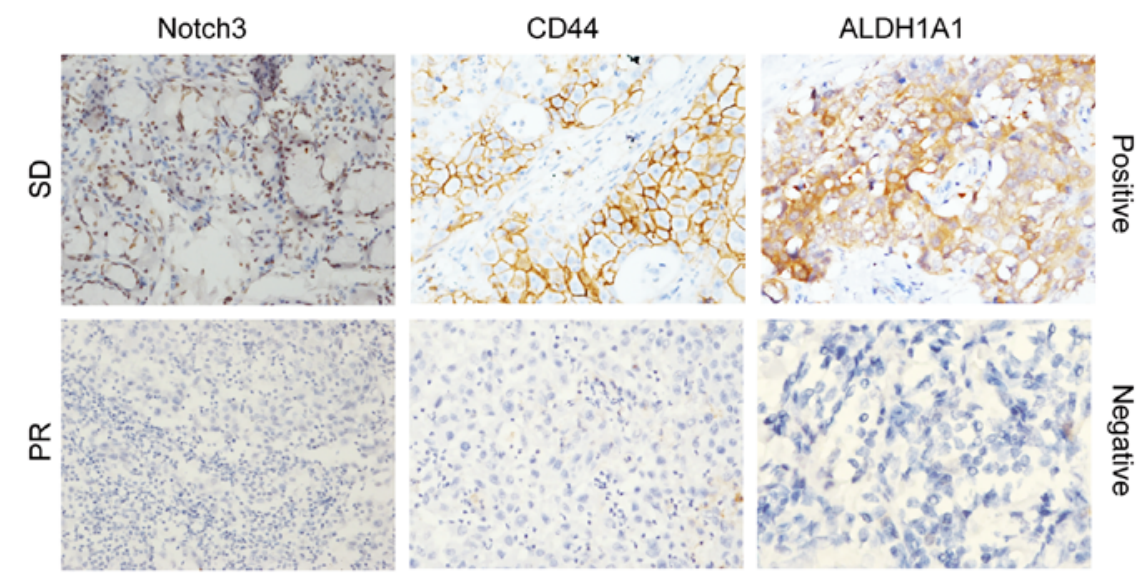

B

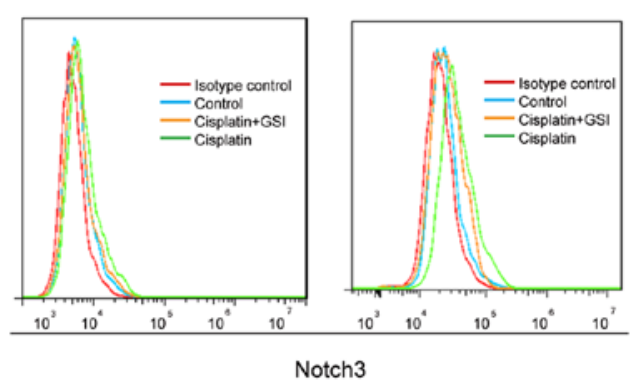

D

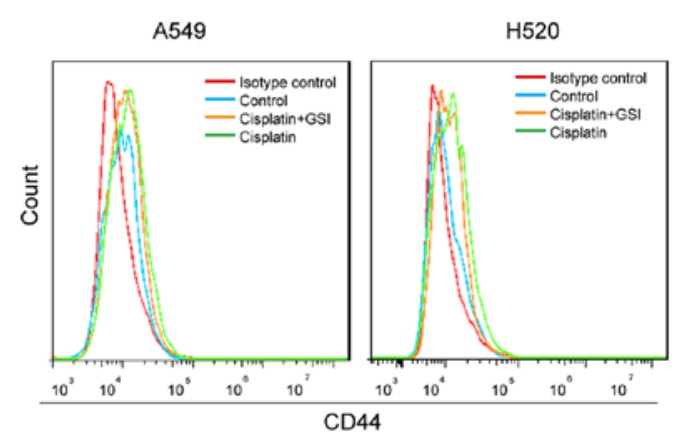

$\mathrm{F}$

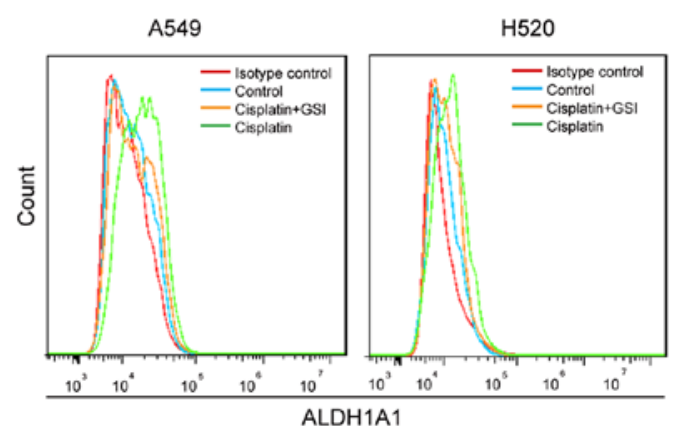

C

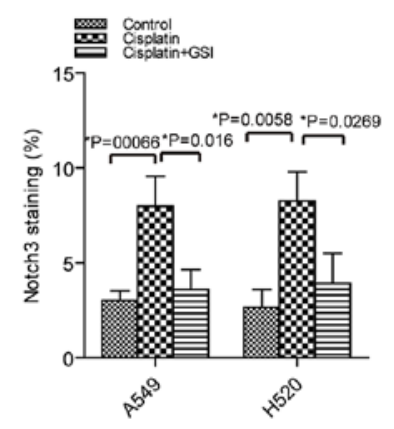

E

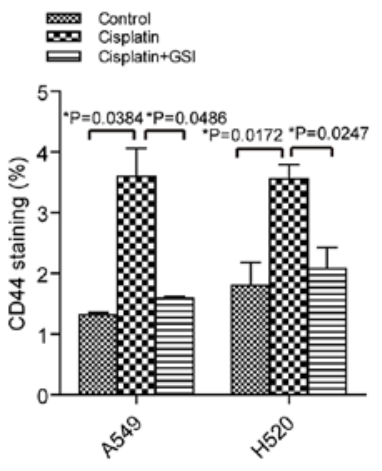

G

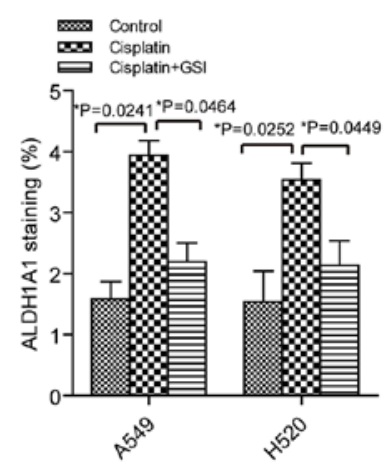

Figure 5. GSI inhibits Notch3, CD44 and ALDH1A1 expression levels in the lung cancer cells with cisplatin treatment. (A) Representative images for Notch3, CD44 and ALDH1A1 staining in primary tumor tissues from the lung cancer patients who received chemotherapy by IHC. (B) Representative images for Notch3 expression in control cells or cells treated with cisplatin or cisplatin plus GSI of A549 and H520 by flow cytometry. (C) Flow cytometry analyses demonstrate that cisplatin increases Notch3 expression, but GSI plus cisplatin decreases this protein expression with significant difference in the lung cancer cells. (D) Representative images for CD44 expression in A549 and H520 cells treated with cisplatin or cisplatin plus GSI by flow cytometry. (E) Flow cytometry analyses show that cisplatin increases CD44 expression, but GSI plus cisplatin decreases this protein expression with significant difference in the A549 and H520 cells. (F) Representative images for ALDH1A1 expression in lung cancer cells stimulated with cisplatin or cisplatin plus GSI by flow cytometry. (G) Histograms show that cisplatin upregulates ALDH1A1 expression, while GSI plus cisplatin downregulate this protein expression with significant difference in lung cancer cells. " $\mathrm{p}<0.05$. 
A

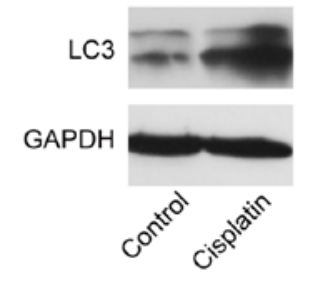

B

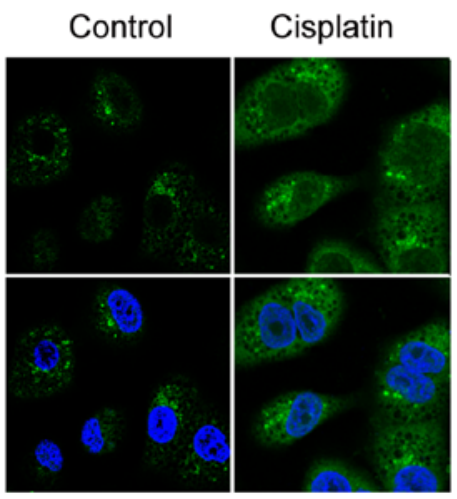

C

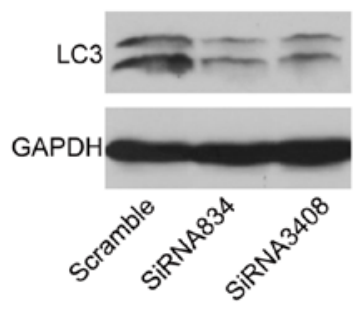

D

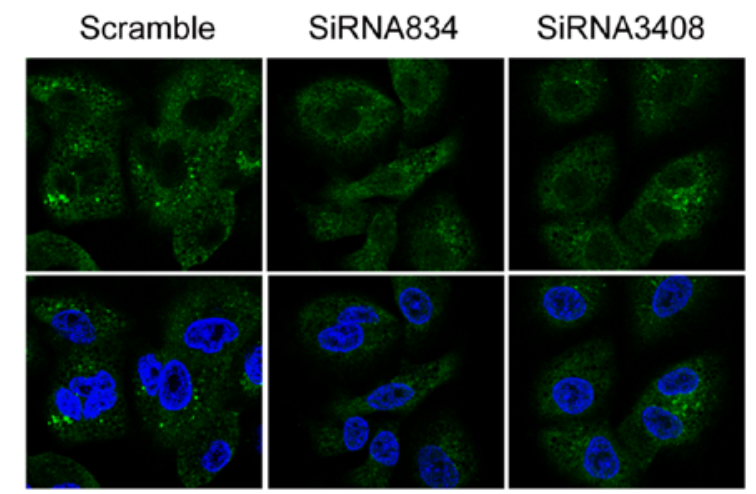

Figure 6. LC3 expression in the A549 cells. (A) The autophagy related marker of LC3 was increased in the cells with cisplatin treatment by western blotting. (B) Immunofluorescence was used to examine LC3 expression in the cells with or without cisplatin. (C) LC3 expression was decreased in the cells transfected with siRNA834 or siRNA3408 compared to the scramble control by western blotting. (D) Immunofluorescence staining shows that both siRNA834 and siRNA3408 transfection inhibits LC3 expression.

not been well elucidated in NSCLC. In the present study, we investigated how Notch3 influenced CSCs to regulate drug resistance in NSCLC cancer cells.

Firstly, we verified that cisplatin could induce colony and sphere formation potential of lung cancer cells A549 and H520. Consistent with a previous study (8), cisplatin improved stemlike characteristics in lung cancer cells. Increasing evidence shows that Notch signal is important in maintenance of tumor cell subpopulation with great clonogenicity of stem-like property in lung cancer $(22,23)$. GSI hinders the final proteolytic cleavage of Notch receptors is a major class of agents inhibiting the Notch pathway. GSI has the ability of anti-cancer growth in several cancer types that regulate CSC property (24-26). We found GSI significantly reduced colony formation and sphere formation potential in the cisplatin treated cells. Our results were consistent with a previous study that GSI inhibited Notch signal restraining cancer cell growth in lung cancer cells (19). Next, we determined whether interference with CSC clonogenicity was through blocking Notch3. Our data showed that specific knock-down of Notch3 led to decrease of colony and sphere formation, indicating Notch3 played an important role in promoting stem-like features in lung cancer cells.

Previous studies have reported several cell surface markers such as CD133, CD166, CD44 and ALDH1A1 that could identify CSCs for NSCLC (11-13). Then, we analyzed the relationship between Notch 3 and these CSC markers and our data showed that Nocth3 expression was positively related to CD44 and ALDH1A1 expression levels in lung cancer tissues. CD44 ${ }^{+}$ cells showed great tumorigenicity by serial tumor transplantation in vivo and higher expression of stem-related factors in the positive lung cancer cells, suggesting CD44 affects the enrichment of stem-like property (27). In lung cancer, $\mathrm{ALDH}^{+}$cells have been proven to be associated with stem-like capacity and suppression of Notch3 could decrease clonogenicity $(17,23)$. In the current study, we clarified that blockade of Notch 3 reduced CD44 and ALDH1A1 expression levels in lung cancer cells. These data indicated that Notch3 may be combined with ALDH1A1 and CD44 to regulate stem-like property in lung cancer cells.

Highly active Notch3 is generally found to be related to poor prognosis in lung cancer patients $(28,29)$. Meta-analysis from Yuan et al showed high activation of Notch3 is linked to poor outcome in lung cancer patients (30). In this metaanalysis, IHC was used to test Notch3 from two articles, and additionally we used qRT-PCR to analyze Notch3 expression in 104 cases of stage I-III NSCLC tissues from Peking University Cancer Hospital. The obtained results showed that high expression of Notch3 is associated with shorter PFS and OS, and Notch3 could be used as a predictor for poor prognosis of NSCLC patients.

It has been documented that Notch signal is involved in drug resistance of cancer cells $(14,31)$. Shi et al demonstrated that overexpression of Notch3 predicts a significantly higher possibility of resistance to chemotherapy in advanced NSCLC patients (32). Currently, we demonstrated that higher expression of Notch3 was shown in the lung cancer tissues from the patients who were resistant to chemotherapy compared to expression of this gene in the tissues from the patients who were sensitive to chemotherapy. Higher expression of CD44 and ALDH1A1 were observed in the lung cancer tissues from 
the NSCLC patients who were resistant to chemotherapy, in comparison to the expression levels of these two markers in the tissues from the NSCLC patients who were sensitive to chemotherapy. Quan et al reported that hyaluronan (HA) is a specific ligand for CD44, and HA-conjugated cisplatin which targets CD44 has highly selective and sensitive anticancer effects in NSCLC cells (33). The novel synthetic curcumin analogues against ALDH1A1 and GSK-3 $\beta$ have functions to overcome chemoresistance in breast cancer (34). Based on induction of the stem-like feature in lung cancer cells by cisplatin treatment, we also observed Notch3, CD44 and ALDH1A1 expression levels were upregulated in the A549 and $\mathrm{H} 520$ cells with cisplatin treatment. Moreover, GSI led to decrease of Notch3, CD44 and ALDH1A1 expression in the lung cancer cells treated with cisplatin to the extent of lung cancer cells without cisplatin treatment. Thus, Notch3 is associated with chemotherapy resistance that might be through CD44 and ALDH1A1.

In general, autophagy refers to an evolutionarily conserved intracellular catabolic process by consisting of several sequential stages: initiation, nucleation, elongation, and maturation to remove damaged cellular components under diverse stressful conditions (35). Autophagy can be conducted as a strategy for cancer cells to overcome the stresses caused by radiation, chemotherapy, or other treatments (20). Therefore, we examined autophagy in lung cancer cells with cisplatin treatment. We observed that autophagy was activated in the lung cancer cells stimulated with cisplatin. In esophageal cancer, chemotherapy was also found to induce autophagy (36), and it has been reported that $\mathrm{cdx} 1$ promoted CSC property that played an essential role in chemoresistance through activation of autophagy in colon cancer (37). We also checked whether Notch3 was related to autophagy and verified that siRNAs of Notch3 inhibited autophagy in lung cancer cells. It was suggested that activation of autophagy is related to chemoresistance of CSCs, a feature that is associated with Notch3 in lung cancer cells.

In conclusion, Notch3 has the capacity to promote colony formation and sphere formation of stem-like capacity in lung cancer cells. After verifying induction of stem-like ability in lung cancer cells with cisplatin, we also found that Notch3 was related to the CSCs markers ALDH1A1 and CD44. Notch3 was shown to be related to poor outcome of NSCLC patients. Moreover, cisplatin promotes autophagy and inhibition of Notch3 was shown to prevent autophagy, providing a novel therapy to target CSCs in lung cancer.

\section{Acknowledgements}

This study was funded by National Natural Science Foundation of China (grant no. 81502578), Peking University (PKU) 985 Special Funding for Collaborative Research with PKU Hospitals, and Beijing Municipal Administration of Hospitals Clinical Medicine Development of special funding support (code: ZYLX201509).

\section{References}

1. Siegel RL, Miller KD and Jemal A: Cancer statistics, 2015. CA Cancer J Clin 65: 5-29, 2015.
2. Lopez-Ayllon BD, Moncho-Amor V, Abarrategi A, Ibañez de Cáceres I, Castro-Carpeño J, Belda-Iniesta C, Perona $\mathrm{R}$ and Sastre L: Cancer stem cells and cisplatin-resistant cells isolated from non-small-lung cancer cell lines constitute related cell populations. Cancer Med 3: 1099-1111, 2014.

3. Nguyen LV, Vanner R, Dirks P and Eaves CJ: Cancer stem cells: An evolving concept. Nat Rev Cancer 12: 133-143, 2012.

4. Eramo A, Lotti F, Sette G, Pilozzi E, Biffoni M, Di Virgilio A, Conticello C, Ruco L, Peschle C and De Maria R: Identification and expansion of the tumorigenic lung cancer stem cell population. Cell Death Differ 15: 504-514, 2008.

5. Ricci-Vitiani L, Lombardi DG, Pilozzi E, Biffoni M, Todaro M, Peschle $\mathrm{C}$ and De Maria R: Identification and expansion of human colon-cancer-initiating cells. Nature 445: 111-115, 2007.

6. Patrawala L, Calhoun T, Schneider-Broussard R, Li H, Bhatia B, Tang S, Reilly JG, Chandra D, Zhou J, Claypool K, et al: Highly purified $\mathrm{CD}_{4} 4^{+}$prostate cancer cells from xenograft human tumors are enriched in tumorigenic and metastatic progenitor cells. Oncogene 25: 1696-1708, 2006.

7. Al-Hajj M, Wicha MS, Benito-Hernandez A, Morrison SJ and Clarke MF: Prospective identification of tumorigenic breast cancer cells. Proc Natl Acad Sci USA 100: 3983-3988, 2003.

8. Liu YP, Yang CJ, Huang MS, Yeh CT, Wu AT, Lee YC, Lai TC, Lee $\mathrm{CH}$, Hsiao YW, Lu J, et al: Cisplatin selects for multidrugresistant $\mathrm{CD} 133^{+}$cells in lung adenocarcinoma by activating Notch signaling. Cancer Res 73: 406-416, 2013.

9. Salnikov AV, Gladkich J, Moldenhauer G, Volm M, Mattern J and Herr I: CD133 is indicative for a resistance phenotype but does not represent a prognostic marker for survival of non-small cell lung cancer patients. Int J Cancer 126: 950-958, 2010.

10. Kitamura H, Okudela K, Yazawa T, Sato H and Shimoyamada H: Cancer stem cell: Implications in cancer biology and therapy with special reference to lung cancer. Lung Cancer 66: 275-281, 2009.

11. Sterlacci W, Savic S, Fiegl M, Obermann E and Tzankov A: Putative stem cell markers in non-small-cell lung cancer: a clinicopathologic characterization. J Thorac Oncol 9: 41-49, 2014.

12. Tachezy M, Zander H, Wolters-Eisfeld G, Müller J, Wicklein D, Gebauer F, Izbicki JR and Bockhorn M: Activated leukocyte cell adhesion molecule (CD166): An 'inert' cancer stem cell marker for non-small cell lung cancer? Stem Cells 32: 1429-1436, 2014.

13. Yan X, Luo H, Zhou X, Zhu B, Wang Y and Bian X: Identification of CD90 as a marker for lung cancer stem cells in A549 and H446 cell lines. Oncol Rep 30: 2733-2740, 2013.

14. Pannuti A, Foreman K, Rizzo P, Osipo C, Golde T, Osborne B and Miele L: Targeting Notch to target cancer stem cells. Clin Cancer Res 16: 3141-3152, 2010.

15. Selkoe D and Kopan R: Notch and Presenilin: Regulated intramembrane proteolysis links development and degeneration. Annu Rev Neurosci 26: 565-597, 2003.

16. Fortini ME: Gamma-secretase-mediated proteolysis in cellsurface-receptor signalling. Nat Rev Mol Cell Biol 3: 673-684, 2002.

17. Hassan KA, Wang L, Korkaya H, Chen G, Maillard I, Beer DG, Kalemkerian GP and Wicha MS: Notch pathway activity identifies cells with cancer stem cell-like properties and correlates with worse survival in lung adenocarcinoma. Clin Cancer Res 19: 1972-1980, 2013.

18. Arasada RR, Amann JM, Rahman MA, Huppert SS and Carbone DP: EGFR blockade enriches for lung cancer stem-like cells through Notch3-dependent signaling. Cancer Res 74: 5572-5584, 2014.

19. Konishi J, Kawaguchi KS, Vo H, Haruki N, Gonzalez A, Carbone DP and Dang TP: Gamma-secretase inhibitor prevents Notch3 activation and reduces proliferation in human lung cancers. Cancer Res 67: 8051-8057, 2007.

20. Hu YL, Jahangiri A, Delay M and Aghi MK: Tumor cell autophagy as an adaptive response mediating resistance to treatments such as antiangiogenic therapy. Cancer Res 72: 4294-4299, 2012.

21. Mathew R, Karantza-Wadsworth V and White E: Role of autophagy in cancer. Nat Rev Cancer 7: 961-967, 2007.

22. Wang Z, Li Y, Banerjee S and Sarkar FH: Emerging role of Notch in stem cells and cancer. Cancer Lett 279: 8-12, 2009.

23. Sullivan JP, Spinola M, Dodge M, Raso MG, Behrens C, Gao B, Schuster K, Shao C, Larsen JE, Sullivan LA, et al: Aldehyde dehydrogenase activity selects for lung adenocarcinoma stem cells dependent on notch signaling. Cancer Res 70: 9937-9948, 2010. 
24. Abel EV, Kim EJ, Wu J, Hynes M, Bednar F, Proctor E, Wang L, Dziubinski ML and Simeone DM: The Notch pathway is important in maintaining the cancer stem cell population in pancreatic cancer. PLoS One 9: e91983, 2014.

25. Grudzien P, Lo S, Albain KS, Robinson P, Rajan P, Strack PR, Golde TE, Miele L and Foreman KE: Inhibition of Notch signaling reduces the stem-like population of breast cancer cells and prevents mammosphere formation. Anticancer Res 30 3853-3867, 2010.

26. Saito N, Fu J, Zheng S, Yao J, Wang S, Liu DD, Yuan Y, Sulman EP, Lang FF, Colman H, et al: A high Notch pathway activation predicts response to $\gamma$ secretase inhibitors in proneural subtype of glioma tumor-initiating cells. Stem Cells 32: 301-312, 2014.

27. Leung EL, Fiscus RR, Tung JW, Tin VP, Cheng LC, Sihoe AD, Fink LM, Ma Y and Wong MP: Non-small cell lung cancer cells expressing CD44 are enriched for stem cell-like properties. PLoS One 5: e14062, 2010.

28. Andersen S, Donnem T, Al-Saad S, Al-Shibli K, Stenvold H Busund LT and Bremnes RM: Correlation and coexpression of HIFs and NOTCH markers in NSCLC. Anticancer Res 31: 1603-1606, 2011.

29. Westhoff B, Colaluca IN, D'Ario G, Donzelli M, Tosoni D, Volorio S, Pelosi G, Spaggiari L, Mazzarol G, Viale G, et al: Alterations of the Notch pathway in lung cancer. Proc Natl Acad Sci USA 106: 22293-22298, 2009.

30. Yuan X, Wu H, Xu H, Han N, Chu Q, Yu S, Chen Y and Wu K: Meta-analysis reveals the correlation of Notch signaling with non-small cell lung cancer progression and prognosis. Sci Rep 5: 10338,2015
31. Wang Z, Li Y, Ahmad A, Azmi AS, Banerjee S, Kong D and Sarkar FH: Targeting Notch signaling pathway to overcome drug resistance for cancer therapy. Biochim Biophys Acta 1806: 258-267, 2010

32. Shi C, Qian J, Ma M, Zhang Y and Han B: Notch 3 protein, not its gene polymorphism, is associated with the chemotherapy response and prognosis of advanced NSCLC patients. Cell Physiol Biochem 34: 743-752, 2014.

33. Quan YH, Kim B, Park JH, Choi Y, Choi YH and Kim HK: Highly sensitive and selective anticancer effect by conjugated HA-cisplatin in non-small cell lung cancer overexpressed with CD44. Exp Lung Res 40: 475-484, 2014.

34. Kesharwani RK, Srivastava V, Singh P, Rizvi SI, Adeppa K and Misra K: A Novel approach for overcoming drug resistance in breast cancer chemotherapy by targeting new synthetic curcumin analogues against aldehyde dehydrogenase 1 (ALDH1A1) and glycogen synthase kinase-3 $\beta$ (GSK-3 $\beta$ ). Appl Biochem Biotechnol 176: 1996-2017, 2015.

35. Green DR and Levine B: To be or not to be? How selective autophagy and cell death govern cell fate. Cell 157: 65-75, 2014.

36. O'Donovan TR, O'Sullivan GC and McKenna SL: Induction of autophagy by drug-resistant esophageal cancer cells promotes their survival and recovery following treatment with chemotherapeutics. Autophagy 7: 509-524, 2011.

37. Wu S, Wang X, Chen J and Chen Y: Autophagy of cancer stem cells is involved with chemoresistance of colon cancer cells. Biochem Biophys Res Commun 434: 898-903, 2013. 